Classification

Physics Abstracts

$02.60-12.35 \mathrm{E}$

\title{
A stabilizing procedure for analytic continuation
}

\author{
S. Ciulli
}

Département de Physique Mathématique, U.S.T.L., Montpellier, France

and T. D. Spearman

School of Mathematics, Trinity College, Dublin 2, Ireland

(Reçu le 2 novembre 1983, révisé le 11 juillet 1984, accepté le 16 juillet 1984)

Résumé. - Une technique de prolongement analytique stable partant d'un ensemble continu de données est décrite sommairement.

Abstract. - A new procedure for stabilizing an analytic continuation off an open curve is described in outline.

Analytic continuation is an important technique in physics. A typical problem involves continuation off an open contour located in the interior of the holomorphy domain, and one may wish to carry this continuation to the boundary. Unfortunately, this problem is arbitrarily unstable in the sense associated with Hadamard, which means that any errors in the input become arbitrarily magnified so that the output is uncontrolled unless some stabilizing condition is imposed.

The boundary of the holomorphy domain may be taken to be the unit circle. The data region, off which the continuation is to be made, is taken to be a segment $\gamma \equiv\left(z_{1}, z_{2}\right)$ of the real axis, where $\left|z_{1}\right|,\left|z_{2}\right|<1$. In this paper we impose a condition based on a smoothness requirement expressed in terms of the derivative $x_{r}(\phi)$ of the imaginary part of the function along the boundary $|z|=1$. The condition which we shall impose is that the following functional should be minimized [1] :

$$
F_{1}\left[x_{\mathrm{r}}\right] \equiv \frac{1}{2 \pi} \int_{0}^{2 \pi}\left(x_{\mathrm{r}}(\phi)\right)^{2} \sigma(\phi) \mathrm{d} \phi .
$$

$\sigma(\phi)$ is some real positive weight function. The complex function $X(z)$ may be reconstructed from the real derivative function $x_{\mathrm{r}}(\phi)$ up to the ambiguity of an additive real constant $d_{0}[2]$.

The problem is to obtain a function $X(z)$ which adequately fits the data in the region $\gamma$ and for which the functional $F_{1}\left[x_{\mathrm{r}}\right]$ has the least possible value. The input data will have errors attached and in terms of these the adequacy of fit of $X(z)$ is expressed in terms of a $\chi^{2}$-function which will be required to have a specified value, say $f \cdot \chi^{2}=f$ may be written as $F_{2}\left[x_{\mathrm{r}} ; d_{0}\right]=0$. This 
minimization is with respect to the function $x_{\mathrm{r}}(\phi)$ and the constant $d_{0}$ and yields a Fredholm equation for $x_{r}(\phi)$.

When the data set $\gamma$ is a finite set of points the integral equation may be reduced to a matrix equation. This discrete problem has already been solved [1] by a quite different method, and one can show [3] that the present method, when applied to the discrete problem, yields the same results as were obtained in reference [1]. The discrete case is appropriate to experimental data but a theoretical input comes naturally in continuous form so that an adequate treatment requires the full analysis outlined above.

When examining the results described in this Letter one essential question must be how effective is the stabilization actually achieved, in particular near the boundary $|z|=1$, when the data $d(x)$ on $\gamma$ vary within their range of errors? This question has been investigated and bounds obtained which will be set out in a forthcoming paper [4].

\section{Acknowledgments.}

Much of the work described in this Letter was carried out at the Institut des Hautes Etudes Scientifiques, Bures-sur-Yvette. Both authors wish to thank the Director, Professor Kuiper, for his hospitality and one of us, S. C., gratefully acknowledges the financial support which he received from the Institut.

\section{References}

[1] Ciulli, S. and Spearman, T. D., Phys. Rev. D 27 (1983) 1580.

[2] Ciulli, S. and Spearman, T. D., J. Math. Phys. 23 (1982) 1752.

[3] Spearman, T. D., invited lecture at the 1983 Smolenice Conference on Hadron Structure - to be published in the Conference Proceedings.

[4] Ciulli, M., Ciulli, S. and Spearman, T. D., to be published in J. Math. Phys. 